\title{
The evolution of global inequality: absolute, relative and intermediate views*
}

\author{
Kristof Bosmans ${ }^{\dagger} \cdot$ Koen Decancq ${ }^{\ddagger}$. André Decoster ${ }^{\ddagger}$ \\ ${ }^{\dagger}$ Department of Economics, Maastricht University, \\ Tongersestraat 53, 6211 LM Maastricht, The Netherlands \\ ${ }^{\ddagger}$ Center for Economic Studies, Katholieke Universiteit Leuven, \\ Naamsestraat 69, 3000 Leuven, Belgium
}

March 30, 2011

\begin{abstract}
We compare absolute, relative and intermediate views on the evolution of global inequality between 1980 and 2009. According to the relative view, inequality remains invariant after a uniform proportional change of all incomes whereas the absolute view requires invariance to a uniform change of all incomes with the same amount. We use a generic intermediate view which states that an income distribution is as unequal as another one if it can be obtained as a weighted average of a uniform proportional and a uniform absolute change of the incomes. Using recent data on GDP per capita for 115 countries, we find considerable support for the claim that world inequality increased for the absolute view and for intermediate views which move substantially in the direction of the relative view.
\end{abstract}

Keywords. Global inequality · Intermediate inequality · Lorenz dominance

JEL classification. D31 · D63 - I31 · O5

\footnotetext{
*We thank Rolf Aaberge, Audun Langørgen and Claudio Zoli for valuable comments. The authors are solely responsible for remaining shortcomings.

E-mail addresses: k.bosmans@maastrichtuniversity.nl (K. Bosmans), koen.decancq@econ.kuleuven.be (K. Decancq), andre.decoster@econ.kuleuven.be (A. Decoster).
} 


\section{Introduction}

In recent years, a substantial empirical literature on global income inequality has emerged. ${ }^{1}$ There is considerable controversy in this literature concerning the evolution of global inequality, with some studies claiming that global inequality has risen over the past few decades and others claiming that it has declined. Since any empirical statement on global inequality rests on both arduous data collection and an inherently normative measurement framework, the reasons for the disagreement can be manifold.

It is well-known that a genuine assessment of global inequality would need micro data on a global scale. In the absence of comparable worldwide income surveys spanning a sufficiently long time period, most authors have fallen back on various techniques to reconstruct yearly global income distributions. As extensively discussed in the survey by Anand and Segal (2008), at least part of the divergence in conclusions across studies is the result of different methodological choices in this construction of global income distributions. However, as far as the measurement apparatus used to assess inequality is concerned, there seems to be a remarkable tacit agreement to focus exclusively on the relative view of inequality, thereby ignoring the a priori equally relevant absolute and intermediate views. Formally, the relative view says that inequality remains invariant if all incomes change by the same proportionate amount, while the absolute view says that inequality remains invariant if all incomes change by the same absolute amount. ${ }^{2}$ When assessing inequality changes against the background of substantial income growth over several decades, as is the case with global inequality, it would be surprising that the specific choice of invariance concept would not have a major impact on the conclusions reached.

Figure 1 illustrates how the choice for absolute or relative invariance may indeed lead to radically different perspectives on the same data. The figure presents the yearly growth in GDP per capita (corrected by PPP factors) between 1980 and 2009 for five quintiles of the 1980 income distribution taking population sizes into account. The left

\footnotetext{
${ }^{1}$ See, for instance, Bourguignon and Morrisson (2002), Capéau and Decoster (2005), Chotikapanich, Valenzuela and Rao (1997), Dowrick and Akmal (2005), Milanovic (2002, 2005), Pinkovskiy and Sala-iMartin (2009) and Sala-i-Martin (2006).

${ }^{2}$ The relative invariance requirement is sometimes interpreted as an innocent technical property to make inequality comparisons independent of the unit in which incomes are measured. But, as several authors have emphasized, this interpretation is problematic (e.g., Kolm, 1976a, pp. 419-420, and Marchant, 2008, pp. 694-695). Invariance requirements deal with the normative issue of how to distribute amounts of various sizes in an inequality-neutral way and hence they reflect an important aspect of the notion of inequality which extends beyond the independence of the unit of measurement. In our empirical application, the issue of the unit of measurement is treated coherently by expressing all incomes in PPP dollars at constant prices.
} 


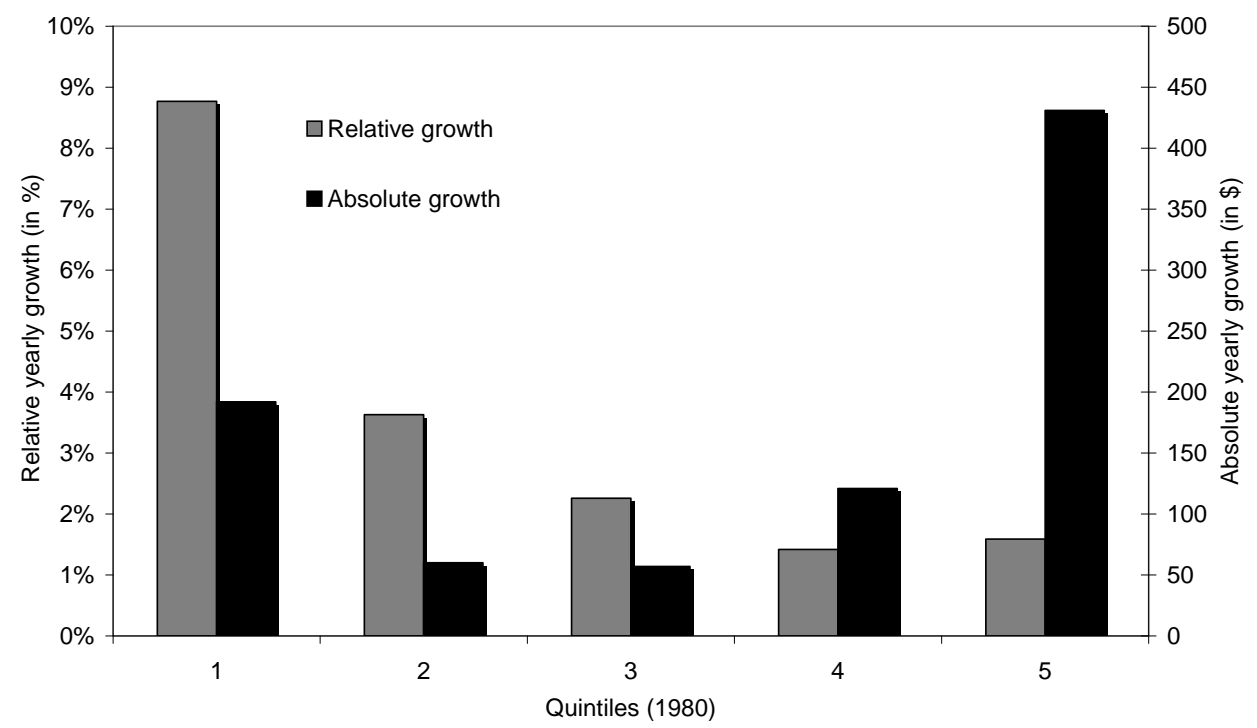

Figure 1: Growth between 1980 and 2009 in PPP-GDP per capita in percentage and in absolute numbers per quintile of the 1980 income distribution (Data: World Bank, 2010)

axis shows the average yearly growth in percentage terms. Obviously, the staggering yearly growth of the bottom quintile, being the fourfold of the growth rates in the upper half of the 1980 distribution, has narrowed the relative gaps between the bottom quintile and the rest of the distribution. The right axis shows the yearly growth of GDP per capita in absolute terms. The comparison between the bottom and the top quintiles is now radically different. Income per capita in the top quintile rose by more than $\$ 431$ per year (or 1.59\%), while that of the bottom quintile only increased by $\$ 192$ per year (or $8.78 \%$ ). This means that the absolute income gap between the bottom and the top quintile has widened. It is clear that conclusions about global income inequality will heavily depend on the particular invariance view adopted.

As also remarked by Ravallion (2003) and Atkinson and Brandolini (2004, 2010), the exclusive focus on the relative view in the study of global income inequality is unwarranted. Already at the beginning of the modern literature on inequality measurement, the various rival invariance views were discussed in seminal contributions by Kolm (1969, 1976a, 1976b). Several concepts of intermediate inequality, each with absolute and relative inequality as polar cases, have since then been proposed - see the comparative studies by Ebert (2004), Zheng (2004) and Zoli (2003) and the references therein. The prevailing appreciation in the theoretical literature is that one cannot argue conclusively in favor of one specific invariance view using only positive arguments. The choice between invari- 
ance views is a matter open to normative disagreement. ${ }^{3}$ Therefore, a complete account of global inequality requires that all alternative invariance views are considered.

In this paper we analyze the evolution of global income inequality by considering absolute and intermediate views in addition to the relative view. We will use the following generic concept of intermediate inequality: inequality remains constant if a rise in total income consists for $\alpha \%$ in an equal proportionate increase and for $(1-\alpha) \%$ in an equal absolute increase. The number $\alpha$ is allowed to vary with the initial distribution and the size of the increase in total income. We show that this generic concept is fully consistent with many of the alternative intermediate concepts that have been developed in the theoretical literature e.g., with those proposed by Besley and Preston (1988), Bossert and Pfingsten (1990), del Rio and Ruiz-Castillo (2000), Krtscha (1994), Pfingsten (1987) and Yoshida (2005). We interpret the parameter $\alpha$ as a measure of relativeness, which quantifies the particular position of an intermediate view between the polar absolute $(\alpha=0)$ and relative $(\alpha=1)$ cases.

We apply this more comprehensive framework to the assessment of global inequality using two perspectives. First, we consider an $\alpha$-intermediate version of the Lorenz dominance criterion. This allows us to determine the ranges of $\alpha$ values for which conclusions hold unanimously for all inequality measures with the corresponding intermediate views. Second, because inequality measures with a common intermediate view do not always unanimously agree, ${ }^{4}$ we also consider $\alpha$-intermediate versions of the S-Gini and generalized entropy classes of inequality measures. We identify the parameter combinations (with one parameter, $\alpha$, measuring the degree of relativeness and the other parameter measuring the degree of bottom-sensitivity) that correspond to the competing judgments on the evolution of global inequality through time. The main contribution of this paper is to provide robust results holding for large classes of inequality measures and to make explicit the dependence of the conclusions on the degree of relativeness. Our analysis is therefore complementary to that of Atkinson and Brandolini (2004, 2010), who focus on particular measures of inequality.

Our empirical analysis uses World Bank (2010) data on population and GDP per capita (in 2005 dollars, corrected using PPPs) for 115 countries in the period 19802009. We make abstraction from within-country inequality. The between-country income distribution consists of the total number of individuals of the 115 countries, with each

\footnotetext{
${ }^{3}$ That people do, in fact, disagree on invariance views has been amply demonstrated using questionnaires. See, e.g., Amiel and Cowell (1999a, 1999b) and Ballano and Ruiz-Castillo (1993).

${ }^{4}$ For instance, although several popular relative inequality measures (such as the Gini and Theil indices) show that global inequality has decreased over time, there are relative inequality measures giving more weight to the top or the bottom of the income distribution that indicate an increase in global inequality (see Capéau and Decoster, 2005).
} 
individual assigned the GDP per capita of his or her country as income (see the appendix for more details on the data). There are two reasons to focus exclusively on the betweencountry component. First, few data are available for within-country distributions on a global scale. Several studies have constructed within-country distributions based on many debatable assumptions, making it difficult to choose objectively among them (see Anand and Segal, 2008). Second, the between-country income distribution constitutes a solid benchmark case. Not only do many of these studies agree that the betweencountry component is the most important component of global income inequality, they also seem to agree on the direction of change of between-country inequality: the relative inequality measures often used in empirical work, such as the Gini and Theil indices, indicate a decline of between-country inequality. It is therefore interesting to see what difference a move to absolute and intermediate measures makes in the particular context of between-country inequality.

The paper proceeds as follows. In Section 2, we outline the generic concept of intermediate inequality used in our analysis and we discuss the links with various existing concepts of intermediate inequality. In Section 3, we consider absolute, intermediate and relative Lorenz dominance comparisons for each pair of income distributions in the sample. Since we have income distributions for each year between 1980 and 2009, there are 435 such pairwise comparisons. For each comparison, we will report the range of values of $\alpha$ for which Lorenz dominance holds. Finally, in Section 4, we consider the same extension of $\alpha$-intermediateness for two classes of inequality measures: the S-Gini and the generalized entropy classes of measures. This allows us to obtain further insights for those cases where there is no $\alpha$-Lorenz dominance. Section 5 concludes.

\section{Absolute, relative and intermediate views}

An income distribution is a vector $x=\left(x_{1}, x_{2}, \ldots, x_{n}\right)$ of positive real numbers with $x_{i}$ the income of individual $i=1,2, \ldots, n$. Incomes are ordered such that $x_{1} \leq x_{2} \leq \cdots \leq x_{n}$. The set $X$ collects all income distributions. The arithmetic mean of $x$ is denoted by $\bar{x}$ and we write $\lambda_{n}$ for the $n$-dimensional vector of which each component is equal to the real number $\lambda$.

An inequality measure is a continuous function $I: X \rightarrow \mathbb{R}$ which satisfies the transfer principle. This principle says that, with total income fixed, a richer-to-poorer transfer decreases inequality. Formally, for each $\left(x_{1}, \ldots, x_{i}, \ldots, x_{j}, \ldots, x_{n}\right)$ in $X$ and each positive real number $\delta$, if $x_{i}<x_{i}+\delta \leq x_{j}-\delta<x_{j}$, then $I\left(x_{1}, \ldots, x_{i}, \ldots, x_{j}, \ldots, x_{n}\right)>$ $I\left(x_{1}, \ldots, x_{i}+\delta, \ldots, x_{j}-\delta, \ldots, x_{n}\right)$.

An invariance view expresses how a change in total income has to be distributed over 
Table 1: Intermediate views as weighted averages of the relative and absolute views

Given distribution $x=(100,200,300,400)$, how should an extra amount $4 \times 250(=4 \times \lambda)$ be divided (resulting in distribution $x^{\prime}$ ) such that inequality is unaffected?

\begin{tabular}{l|r|c|r|r|r}
\hline Inequality view & \multicolumn{1}{|c|}{$\alpha$} & $x^{\prime}$ & $\mu_{B P}$ & $\mu_{P}$ & $\mu_{Y}$ \\
\hline Absolute & 0 & $(350,450,550,650)$ & $\infty$ & 0 & 0 \\
Intermediate & 0.10 & $(335,445,555,665)$ & 2250 & 0.00044 & 0.138 \\
Intermediate & 0.20 & $(320,440,560,680)$ & 1000 & 0.00100 & 0.263 \\
Intermediate & 0.50 & $(275,425,575,725)$ & 250 & 0.00398 & 0.585 \\
Intermediate & 0.80 & $(230,410,590,770)$ & 62.5 & 0.01575 & 0.848 \\
Intermediate & 0.90 & $(215,405,595,785)$ & 27.7 & 0.03475 & 0.926 \\
Relative & 1 & $(200,400,600,800)$ & 0 & 1 & 1 \\
\hline
\end{tabular}

the individuals such that inequality remains invariant. Suppose distribution $x^{\prime}$ differs from distribution $x$ by an extra amount of income $n \lambda$, or $\bar{x}^{\prime}=\bar{x}+\lambda$. According to the relative invariance view, income distribution $[(\bar{x}+\lambda) / \bar{x}] x$ shows the same inequality as income distribution $x$, i.e., inequality remains the same if all incomes are multiplied by the same factor $\bar{x}^{\prime} / \bar{x}$. According to the absolute invariance view, income distribution $x+\lambda_{n}$ shows the same inequality as income distribution $x$, i.e., inequality remains the same if all incomes are increased by the same amount $\lambda$. We consider intermediate views that are weighted averages of the relative and absolute views. That is, according to an intermediate inequality view, for every distribution $x$ and for every amount of per capita growth $\lambda$, there is an $\alpha$ between 0 and 1 such that distribution $x^{\prime}$, defined as

$$
x^{\prime}=\alpha \frac{\bar{x}+\lambda}{\bar{x}} x+(1-\alpha)\left(x+\lambda_{n}\right),
$$

is equally unequal as distribution $x$. The cases $\alpha=0$ and $\alpha=1$ correspond to the absolute and relative views, respectively. Intermediate views may be closer or further to each of these extremes in varying degrees. Because $\alpha$ in equation (1) depends on $x$ and on $\lambda$, we consider $\alpha$ as a local measure of the degree of relativeness.

Table 1 gives an example. The initial distribution is $x=(100,200,300,400)$. A per capita increase in income of $\lambda=250$ amounts to a doubling of average income from $\bar{x}=250$ to $\bar{x}^{\prime}=500$. The first row of the table corresponds to the absolute view, where all individuals receive the same absolute increase in income of 250 , whereas the last row corresponds to the relative view, where all incomes are doubled. The rows in between show the distributions $x^{\prime}$ corresponding to values of $\alpha$ between 0 and 1 .

Most concepts of intermediate inequality suggested in the literature use the weighted average form of equation (1) and, hence, are covered by our general approach. The difference between the various concepts of intermediate inequality lies in how $\alpha$ varies 
with the mean of $x$ and with the size of $\lambda$. For the concept of Pfingsten (1987), $\alpha$ equals $\mu_{P} \bar{x} /\left(\mu_{P} \bar{x}+1-\mu_{P}\right)$ with $\mu_{P}$ a constant between 0 and $1 .{ }^{5}$ For that of Besley and Preston (1988), $\alpha$ equals $\bar{x} /\left(\bar{x}+\mu_{B P}\right)$ with $\mu_{B P}$ a constant greater than $0 .{ }^{6}$ For the concept of del Rio and Ruiz-Castillo (2000), $\alpha$ is a constant. ${ }^{7}$ Finally, for that of Yoshida (2005), $\alpha$ equals $\left[\left(\frac{\bar{x}+\lambda}{\bar{x}}\right)^{\mu_{Y}}-1\right] /\left(\frac{\bar{x}+\lambda}{\bar{x}}-1\right)$ with $\mu_{Y}$ a constant between 0 and $1 .^{8}$ The choice of the specific parameter value, $\mu_{P}, \mu_{B P}$ or $\mu_{Y}$, determines an $\alpha$ for every $x$ and every $\lambda$. Moreover, $\alpha$ is a strictly monotonic function of $\mu_{P}, \mu_{B P}$ and $\mu_{Y}$. In other words, $\alpha$ is a natural way of measuring relativeness for each of these concepts of intermediate inequality. In the three rightmost columns of Table 1 we give the parameter values for the various concepts that correspond to the given values of $\alpha$.

\section{$3 \alpha$-Lorenz dominance}

The chosen invariance view only determines iso-inequality loci of distributions with different total incomes. To broaden the scope of inequality comparisons, we now combine the invariance requirement with the transfer principle, as embodied in the Lorenz dominance criterion.

For income distributions $x$ and $y$ with equal means, $x$ is said to Lorenz dominate $y$ if

$$
\sum_{i=1}^{k} x_{i} \geq \sum_{i=1}^{k} y_{i} \quad \text { for all } k=1,2, \ldots, n-1,
$$

with at least one inequality holding strictly. ${ }^{9}$ Above, we have defined an inequality measure as satisfying the transfer principle. It is well-known that $x$ Lorenz dominates $y$ if and only if all inequality measures agree that $x$ is strictly less unequal than $y$.

Now suppose that the income distributions $x$ and $y$ do not have equal means. Then we need a prior step to equalize the means and it is here that the particular invariance view comes into play. Suppose $\bar{x}<\bar{y}$. We then look for an income distribution $x_{\alpha}$ that is equally unequal as $x$ but has a mean equal to that of $y$. Suppose that $\alpha$ is the local

\footnotetext{
${ }^{5}$ This concept is further explored by Bossert and Pfingsten (1990).

${ }^{6}$ This concept is based on that of Kolm (1976b).

${ }^{7}$ We present here a variant of the concept of del Rio and Ruiz-Castillo (2000). This variant is a special case of the concept proposed by Seidl and Pfingsten (1997) which was in turn inspired by Ballano and Ruiz-Castillo (1993). The very broad concept of Seidl and Pfingsten (1997) does not in general fit into the framework presented here.

${ }^{8}$ Yoshida (2005) generalizes Krtscha (1994). In the latter concept $\mu_{Y}$ equals 0.5.

${ }^{9}$ The Lorenz dominance criterion is defined here for the fixed population case. For our empirical analysis we extend this definition, as is common, using the replication invariance principle. This principle says that inequality remains the same if the income distribution is replicated any number of times. Note that the inequality measures considered in Section 4 satisfy replication invariance by definition.
} 
degree of relativeness we want to impose. Then we consider

$$
x_{\alpha}=\alpha \frac{\bar{y}}{\bar{x}} x+(1-\alpha)\left(x+(\bar{y}-\bar{x})_{n}\right) .
$$

By construction $x_{\alpha}$ and $y$ have equal means and hence can be compared using Lorenz dominance. This procedure leads to the following concept of $\alpha$-Lorenz dominance.

Definition 1. Let $x$ and $y$ be two income distributions such that $\bar{x} \leq \bar{y}$. Let $x_{\alpha}$ be defined as in equation (2). We say that $x \alpha$-Lorenz dominates $y$ if $x_{\alpha}$ Lorenz dominates $y$. We say that $y \alpha$-Lorenz dominates $x$ if $y$ Lorenz dominates $x_{\alpha} \cdot{ }^{10}$

Note that $\alpha$-Lorenz dominance of $x$ over $y$ is equivalent to agreement of all inequality measures with a local relativeness of $\alpha$-i.e., all measures according to which $x$ is equally unequal as $x_{\alpha}$ - that $x$ is less unequal than $y$. Furthermore, 1-Lorenz dominance and 0Lorenz dominance correspond to relative and absolute Lorenz dominance, respectively. ${ }^{11}$

Stronger judgments may however be deduced from the fact that $x \alpha$-Lorenz dominates $y$, depending on whether $x$ or $y$ has the higher mean. Consider again the example of Table 1 with $x=(100,200,300,400)$. Let $y=(220,450,580,750)$, a distribution with twice the total income of $x$. Note that $x_{0.5}=(275,425,575,725)$ Lorenz dominates $y$, and hence that $x$ 0.5-Lorenz dominates $y$. One easily checks in Table 1 that we also have $\alpha$-Lorenz dominance of $x$ over $y$ for all values of $\alpha$ smaller than 0.50 ; that means that all inequality measures with a local relativeness of 0 (absolute) up to 0.50 will agree that inequality has increased in the transition from $x$ to $y$. On the other hand, note that $y$ 0.9-Lorenz dominates $x$ since $y=(220,450,580,750)$ Lorenz dominates $x_{0.9}=(215,405,595,785)$. Once again, one easily checks that $y$ also $\alpha$-Lorenz dominates $x$ for all $\alpha$ greater than 0.90. Hence, all inequality measures with a local relativeness of 0.90 or more would agree that inequality has decreased in the transition from $x$ to $y$. Note that neither $x_{0.8}$ Lorenz dominates $y$, nor $y$ Lorenz dominates $x_{0.8} \cdot{ }^{12}$ See del Rio and Ruiz-Castillo (2000, pp. 232-233) for a related discussion.

The logic of the numerical example can be generalized to the following result.

\footnotetext{
${ }^{10}$ As we saw in Section 2, the $\alpha$-intermediate view is consistent with many of the invariance concepts proposed in the theoretical literature. By consequence, $\alpha$-Lorenz dominance is also consistent with Lorenz dominance criteria based on those invariance concepts. Consider as an example the case of Pfingsten's (1987) concept of inequality invariance: by substituting $\alpha=\mu \bar{x} /(\mu \bar{x}+1-\mu)$ in the expression $\sum_{i=1}^{k} \alpha\left(\bar{y} x_{i} / \bar{x}\right)+(1-\alpha)\left(x_{i}+\bar{y}-\bar{x}\right) \geq \sum_{i=1}^{k} y_{i}$, we obtain the condition of Pfingsten's (1986) Lorenz dominance criterion, viz., $\sum_{i=1}^{k}\left(x_{i}-\bar{x}\right) /(\mu \bar{x}+1-\mu) \geq \sum_{i=1}^{k}\left(y_{i}-\bar{y}\right) /(\mu \bar{y}+1-\mu)$.

${ }^{11}$ See Moyes (1987) for a comparison of relative and absolute Lorenz dominance. This paper introduced the absolute Lorenz dominance criterion into the literature.

${ }^{12}$ To be more precise, it can be shown that $x \alpha$-Lorenz dominates $y$ for every $\alpha$ in the interval $[0,0.514]$ and that $y \alpha$-Lorenz dominates $x$ for every $\alpha$ in the interval $[0.867,1]$. For other values of $\alpha$, the distributions $x$ and $y$ are not Lorenz comparable.
} 
Proposition 1. Let $x$ and $y$ be two income distributions such that $\bar{x} \leq \bar{y}$. If $x \hat{\alpha}$-Lorenz dominates $y$, then $x \alpha$-Lorenz dominates $y$ for all $\alpha<\hat{\alpha}$. If $y \tilde{\alpha}$-Lorenz dominates $x$, then $y \alpha$-Lorenz dominates $x$ for all $\alpha>\tilde{\alpha}$.

Proof. We only prove the case where $x \hat{\alpha}$-Lorenz dominates $y$ since the proof of the other case is very similar. Let $x \hat{\alpha}$-Lorenz dominate $y$. Let $\alpha<\hat{\alpha}$. We need to show that if $\bar{x}<\bar{y}$, then we have $\sum_{i=1}^{k} \alpha \frac{\bar{y}}{\bar{x}} x_{i}+(1-\alpha)\left(x_{i}+\bar{y}-\bar{x}\right) \geq \sum_{i=1}^{k} \hat{\alpha} \frac{\bar{y}}{\bar{x}} x_{i}+(1-\hat{\alpha})\left(x_{i}+\bar{y}-\bar{x}\right)$ for all $k=1,2, \ldots, n-1$. We rewrite the requirement as $(\hat{\alpha}-\alpha) \sum_{i=1}^{k}\left(x_{i}+\bar{y}-\bar{x}\right) \geq$ $(\hat{\alpha}-\alpha) \sum_{i=1}^{k} \frac{\bar{y}}{\bar{x}} x_{i}$. Letting $\bar{y}=(1+\lambda) \bar{x}$, this becomes $\sum_{i=1}^{k}\left(x_{i}+\lambda \bar{x}\right) \geq \sum_{i=1}^{k}\left(x_{i}+\lambda x_{i}\right)$. We get $\lambda k \bar{x} \geq \lambda \sum_{i=1}^{k} x_{i}$ for all $k=1,2, \ldots, n-1$, which is indeed true since $\lambda>0$.

We tested $\alpha$-Lorenz dominance for all 435 possible pairwise comparisons of the world income distribution for the years between 1980 and 2009. In view of Proposition 1, we need only present, for every pair of world income distributions $x$ and $y$ with $\bar{x} \leq \bar{y}$, the maximal $\hat{\alpha}$ for which $x \alpha$-Lorenz dominates $y$ and the minimal $\tilde{\alpha}$ for which $y \alpha$-Lorenz dominates $x$. We start with the former, given in Table 2. For expositional convenience, the table presents the years in order of increasing average income. Not surprisingly, average world GDP per capita increased in almost every year between 1980 and 2009. The only two exceptions are 1982 (decrease of $1.2 \%$ ) and 2009 (decrease of $1.5 \%$ ). ${ }^{13}$ Each cell of Table 2 gives the maximal $\hat{\alpha}$ for which the row year $\alpha$-Lorenz dominates the column year. This means that the distribution obtained by $\hat{\alpha}$-rescaling the row year distribution upwards, Lorenz dominates the column year distribution. ${ }^{14}$ The $\alpha$-Lorenz ordering being a partial ordering, we report '-' if there is no value of $\alpha$ such that the row year $\alpha$-Lorenz dominates the column year.

To interpret Table 2, we first focus on a typical case where average income increased through time. Consider the change in the world income distribution between 1980 and 2005. We have $\hat{\alpha}=0.46$, which means that $1980 \alpha$-Lorenz dominates 2005 for $\alpha$ equal to 0.46 and, using Proposition 1, also for all values of $\alpha$ between 0 and 0.46 . That is, all inequality measures with a local relativeness between 0 (absolute) and 0.46 agree that inequality has increased between 1980 and 2005. Of the 435 possible comparisons, there exists a critical $\hat{\alpha}$ in 215 cases. For these 215 dominance cases, the median value of $\hat{\alpha}$ equals 0.48 and the mean value equals 0.46 . Only two out of these 215 critical $\hat{\alpha}$

\footnotetext{
${ }^{13}$ Although average income increased between 1982 and 1983 (from $\$ 5,944$ to \$5,990), it was still lower in 1983 than in 1981 (when it was $\$ 6,015$ ). Only in 1984 the income level $(\$ 6,156)$ again exceeded the level of 1981 . Hence, for the years 1981 to 1983 , we have $\bar{x}_{1982}<\bar{x}_{1983}<\bar{x}_{1980}<\bar{x}_{1981}<\bar{x}_{1984}$. For the recession of 2009 , we have $\bar{x}_{2006}<\bar{x}_{2009}<\bar{x}_{2007}<\bar{x}_{2008}$.

${ }^{14}$ We compared the Lorenz-ordinates at each point of the union of abscissa points defined by the corners of the stepwise distribution functions of $x$ and $y$.
} 


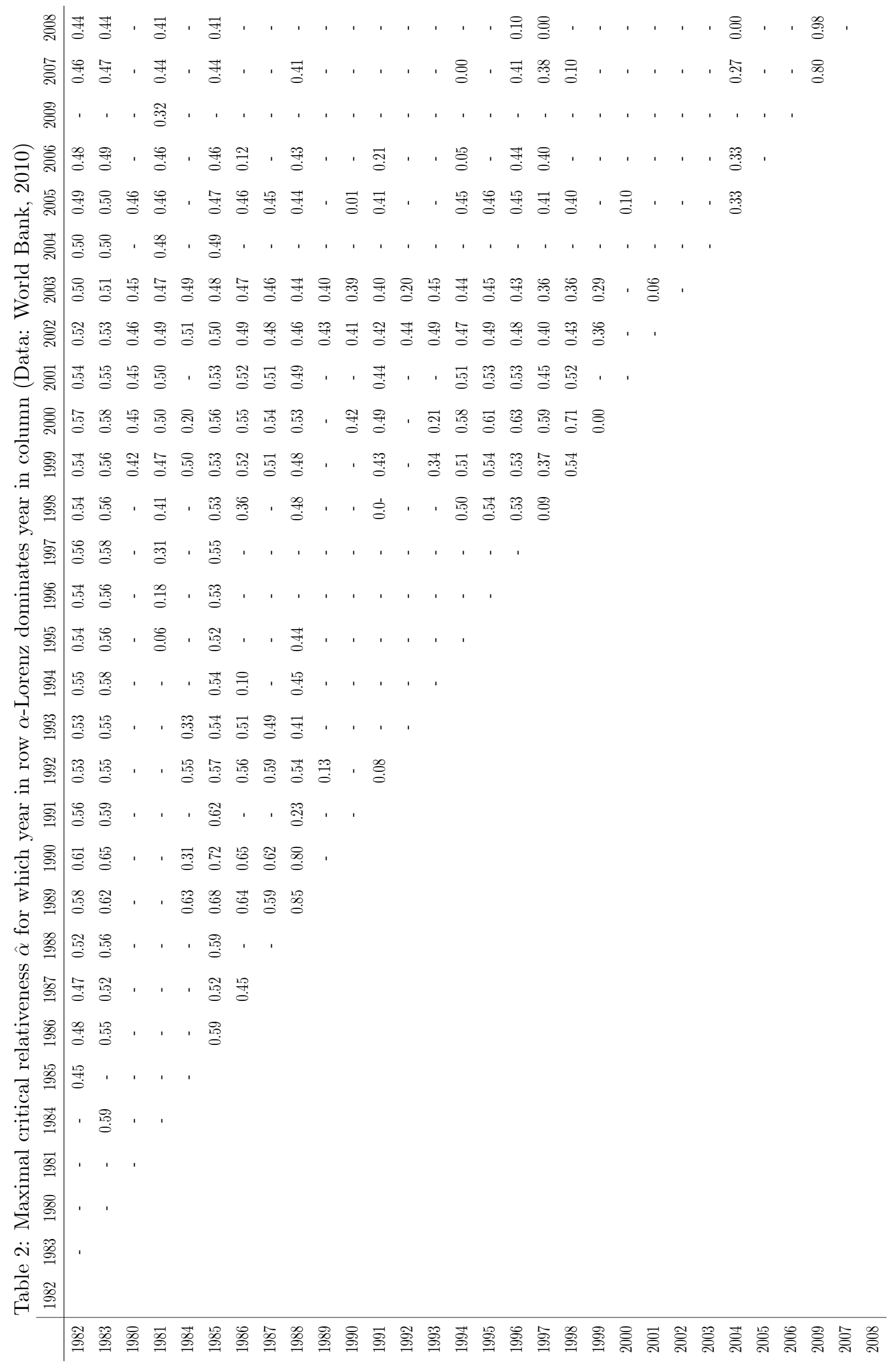


values involve a comparison which runs back in time (viz., the comparison of 2009 with respectively 2007 and 2008). Therefore, we conclude that Table 2 gives considerable support for the claim that world inequality has increased through time for the absolute view, and also for invariance views which move substantially in the direction of the relative view.

Comparisons involving the year 2009 deserve a special mention. First, the comparison of the world income distribution of 2009 with that of 2008 (where 2008 has the higher average income) yields a critical $\hat{\alpha}=0.98$. This means that the statement that inequality has decreased between 2008 and the recession year 2009 is very robust. The pattern of for many countries, negative - growth rates in 2009 must have been such that, roughly speaking, both the absolute gaps narrowed and the ratios shrunk. Second, it is remarkable that the column labeled '2009' contains only one number, viz., for the row '1981'. This means that, with the exception of 1981, we cannot claim that previous years were more equal than 2009, not even if we take the absolute view.

The results of Table 2 present non-negligible evidence for the claim that inequality has increased over the last thirty years if one takes a sufficiently absolute view. This conclusion is not incompatible with a claim that inequality has decreased provided one takes a sufficiently relative view. Therefore, we also computed the minimal $\tilde{\alpha}$ values such that a column year (in Table 2) $\alpha$-Lorenz dominates a row year. However, we do not present the table with $\tilde{\alpha}$ values. The reason is simple: for none of the 435 bilateral comparisons we found an $\alpha$-Lorenz dominance of a year with a higher mean income over a year with a lower mean income. By consequence - with the few exceptions of decreasing income through time - we can safely conclude that one cannot claim that global inequality decreased through time for all inequality measures with a given minimal level of relativeness. Even if we restrict attention to the relative measures alone, we cannot make the statement that between-country inequality unambiguously decreased for all these measures. This means that the common finding in the literature that relative between-country inequality has decreased through time is dependent on the particular subset of relative inequality measures used. In the next section we have a closer look at the conclusions for specific inequality measures.

\section{Inequality measures}

If the $\alpha$-Lorenz dominance criterion fails to yield a conclusion, this means that at least some inequality measures do not agree on the direction of the inequality change. In this case it is interesting to consider specific inequality measures separately in order to obtain further insights. We consider two classes of inequality measures: the S-Gini class and 
the generalized entropy class. We first define the relative cases of the two classes and then consider extensions that also include absolute and intermediate measures.

The first class we consider is that of the S-Gini inequality measures (Donaldson and Weymark, 1980, 1983). Recall that $i$ is the rank position of individual $i$ in the income distributions (since incomes are ordered such that $x_{1} \leq x_{2} \leq \cdots \leq x_{n}$ ). For the relative case, we have

$$
S G_{\rho}(x)=1-\sum_{i=1}^{n}\left[\left(\frac{n-i+1}{n}\right)^{\rho}-\left(\frac{n-i}{n}\right)^{\rho}\right] \frac{x_{i}}{\bar{x}}, \quad \rho \geq 1 .
$$

Second, we consider the class of generalized entropy inequality measures (Bourguignon, 1979, Cowell, 1980, Cowell and Kuga, 1981a, 1981b, Shorrocks, 1980, 1984). For the relative case, we have

$$
G E_{\gamma}(x)= \begin{cases}\frac{1}{n\left(\gamma^{2}-\gamma\right)} \sum_{i=1}^{n}\left[\left(\frac{x_{i}}{\bar{x}}\right)^{\gamma}-1\right] & \text { for } \gamma \neq 0,1, \\ \frac{1}{n} \sum_{i=1}^{n} \ln \left(\frac{\bar{x}}{x_{i}}\right) & \text { for } \gamma=0 \\ \frac{1}{n} \sum_{i=1}^{n}\left(\frac{x_{i}}{\bar{x}}\right) \ln \left(\frac{x_{i}}{\bar{x}}\right) & \text { for } \gamma=1 .\end{cases}
$$

The parameter $\rho$ in the S-Gini class and the parameter $\gamma$ in the generalized entropy class both measure the degree of bottom-sensitivity. The higher the value of $\rho$, or the lower the value of $\gamma$, the more weight is given to transfers at the bottom of the distribution relative to transfers at the top. ${ }^{15}$ Some familiar inequality measures are obtained for specific values of the parameters. Setting $\rho=2$ in the S-Gini class gives the Gini index. For the generalized entropy class, setting $\gamma<1$ gives measures which are ordinally equivalent to the class of measures proposed by Atkinson (1970), setting $\gamma=0$ gives the mean logarithmic deviation and setting $\gamma=1$ gives the Theil inequality measure.

The two classes given in equations (3) and (4) are relative. We use the same approach as in the previous section with Lorenz dominance to allow for all degrees of relativeness between 0 and 1 .

Definition 2. Let $x$ and $y$ be two income distributions such that $\bar{x} \leq \bar{y}$. Let $x_{\alpha}$ be defined as in equation (2). We say that $x$ is at least as unequal as $y$ according to the $(\alpha, \rho)$-S-Gini inequality measure if $S G_{\rho}\left(x_{\alpha}\right) \geq S G_{\rho}(y)$. We say that $y$ is at least as unequal as $x$ according to the $(\alpha, \rho)$-S-Gini inequality measure if $S G_{\rho}(y) \geq S G_{\rho}\left(x_{\alpha}\right)$.

It is tempting to extend the class of generalized entropy measures in equation (4) analogously. This would read as follows.

\footnotetext{
${ }^{15}$ With $\rho$ sufficiently high or $\gamma$ sufficiently low, the focus is on the smallest income as a fraction of the mean. With $\gamma$ sufficiently high, the focus is on the highest income as a fraction of the mean.
} 
Definition 3. Let $x$ and $y$ be two income distributions such that $\bar{x} \leq \bar{y}$. Let $x_{\alpha}$ be defined as in equation (2). We say that $x$ is at least as unequal as $y$ according to the $(\alpha, \gamma)$-generalized entropy inequality measure if $G E_{\gamma}\left(x_{\alpha}\right) \geq G E_{\gamma}(y)$. We say that $y$ is at least as unequal as $x$ according to the $(\alpha, \gamma)$-generalized entropy inequality measure if $G E_{\gamma}(y) \geq G E_{\gamma}\left(x_{\alpha}\right)$.

However, contrary to the criterion in Definition 2, the criterion in Definition 3 is not transitive. ${ }^{16}$ For example, consider the measure with parameter values $\alpha=0$ and $\gamma=1$ and the income distributions $x=(10,30,50), y=(14,22,54), x^{\prime}=x+(10,10,10)=$ $(20,40,60)$ and $y^{\prime}=y+(10,10,10)=(24,32,64)$. Since the $(0,1)$-generalized entropy measure is absolute, distributions $x$ and $x^{\prime}$ are equally unequal and the same goes for distributions $y$ and $y^{\prime}$. Because distributions $x$ and $y$ have equal means, Definition 3 demands we follow the judgment of the relative generalized entropy measure with $\gamma=1$. The same is true for the distributions $x^{\prime}$ and $y^{\prime}$. We have $G E_{1}(x)>G E_{1}(y)$ and $G E_{1}\left(x^{\prime}\right)<G E_{1}\left(y^{\prime}\right)$, and hence $x$ is more unequal than $y$ and $x^{\prime}$ is less unequal than $y^{\prime}$. We obtain the following cycle: $x^{\prime}$ is equally unequal as $x, x$ is more unequal than $y, y$ is equally unequal as $y^{\prime}$ and $y^{\prime}$ is more unequal than $x^{\prime}$. Although with our focus on isolated pairwise comparisons in the empirical application, this lack of transitivity is not really a problem, we still prefer to work with a transitive extension of the generalized entropy class of inequality measures. We therefore use the method of Bossert and Pfingsten (1990) to extend the relative class in equation (4).

Definition 4. Let $x$ and $y$ be two income distributions such that $\bar{x} \leq \bar{y}$. We say that $x$ is at least as unequal as $y$ according to the Bossert-Pfingsten $(\alpha, \gamma)$-generalized entropy inequality measure if $G E_{\gamma}\left(x+[(1-\mu) / \mu]_{n}\right) \geq G E_{\gamma}\left(y+[(1-\mu) / \mu]_{n}\right)$ with $\alpha=\mu \bar{x} /(\mu \bar{x}+1-\mu)$. We say that $y$ is at least as unequal as $x$ according to the Bossert-Pfingsten $(\alpha, \gamma)$-generalized entropy inequality measure if $G E_{\gamma}\left(y+[(1-\mu) / \mu]_{n}\right) \geq$ $G E_{\gamma}\left(x+[(1-\mu) / \mu]_{n}\right)$ with $\alpha=\mu \bar{x} /(\mu \bar{x}+1-\mu) \cdot{ }^{17}$

Figures 2 and 3 summarize the empirical results. Each bilateral comparison between two selected years is represented by a demarcation curve in the two-parameter space of respectively $(\alpha, \rho)$ for the S-Gini class and $(\alpha, \gamma)$ for the generalized entropy class. The curve depicts the parameter combinations for which inequality between the two years

\footnotetext{
${ }^{16}$ In fact, the criterion in Definition 2 is equivalent to the Bossert and Pfingsten (1990, p. 132) extension of the Gini indices. That is, for all income distributions $x$ and $y$, we have that $x$ is at least as unequal as $y$ according to the $(\alpha, \rho)$-S-Gini inequality measure if and only if $\bar{x} S G_{\rho}(x) /(\mu \bar{x}+1-\mu) \geq \bar{y} S G_{\rho}(y) /(\mu \bar{y}+$ $1-\mu)$, with $\alpha=\mu \bar{x} /(\mu \bar{x}+1-\mu)$ if $\bar{x} \leq \bar{y}$ and $\alpha=\mu \bar{y} /(\mu \bar{y}+1-\mu)$ if $\bar{x} \geq \bar{y}$.

${ }^{17}$ Cowell (2006) discusses how the absolute case is obtained by taking the limit $(1-\mu) / \mu \rightarrow \infty$. This absolute subclass has also been characterized axiomatically — see Chakravarty and Tyagarupananda (1998, 2009) and Bosmans and Cowell (2010).
} 


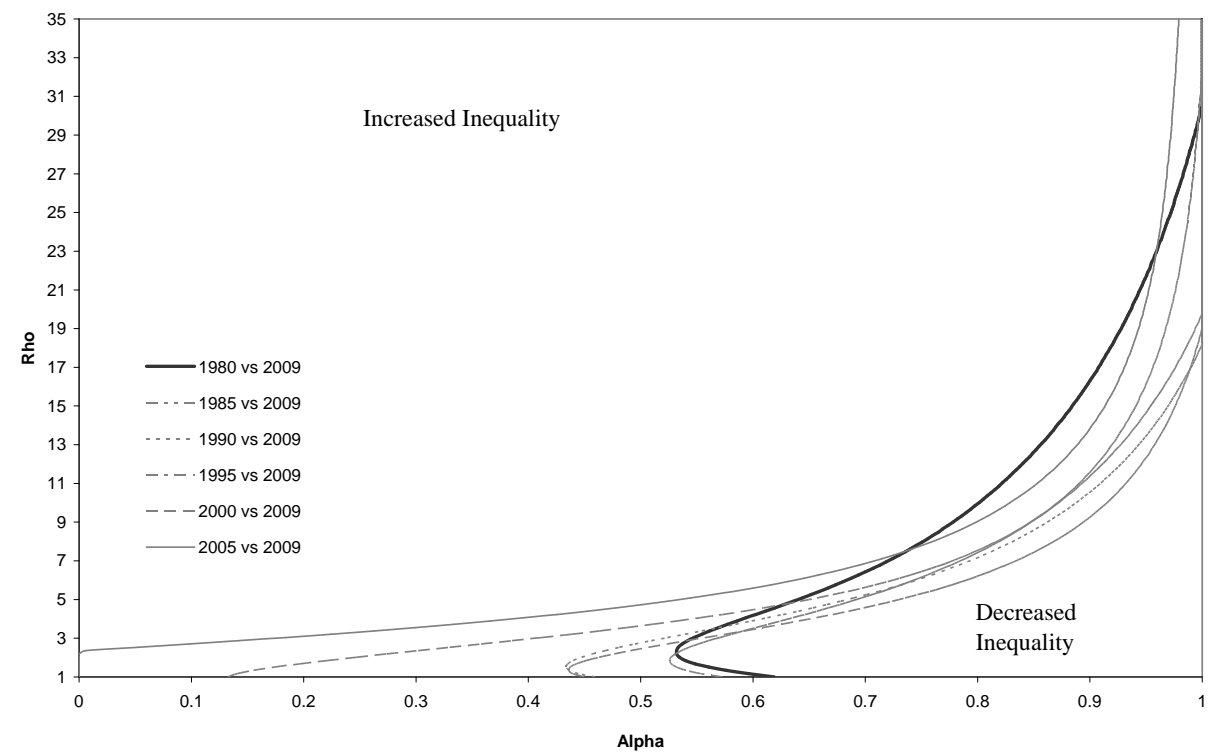

Figure 2: Increasing and decreasing inequality in the $(\alpha, \rho)$ normative space according to the S-Gini index (Data: World Bank, 2010)

remains unchanged. For the parameter combinations to the left of these iso-inequality lines inequality has increased over time, while for the combinations to the right it has decreased. Since the figures would become illegible if we present all bilateral comparisons, we made a selection for some relevant subperiods.

Figure 2 reveals that the members of the S-Gini class unanimously agree that inequality has increased between 1980 and 2009 for values of $\alpha$ up to 0.532 . This means that one has to stick to a local relativeness of at least 0.532 if one wants to conclude that inequality decreased over the thirty year period between 1980 and 2009. The greater the focus on the more bottom-sensitive members of the class (corresponding to higher values of $\rho$ ), the less one has to go in the direction of the absolute view to conclude that inequality has increased. S-Ginis measures with sufficiently high values of $\rho$ even agree that inequality has increased irrespective of the level of local relativeness $\alpha$. If we shorten the period of comparison (always comparing with the final year 2009), the pattern remains the same: an increase in $\rho$ pushes the minimal $\alpha$ for which we conclude that inequality increased upwards.

Figure 3 shows that the generalized entropy inequality measures agree on increased inequality between 1980 and 2009 for values of $\alpha$ up to 0.492. This minimal value of $\alpha$ occurs at a bottom-sensitivity of $\gamma=-3$, but for other values of $\gamma$ one can afford to take a less absolute view and still conclude that global inequality has increased. Similar to the S-Gini class, even the pure relative members of the generalized entropy class 


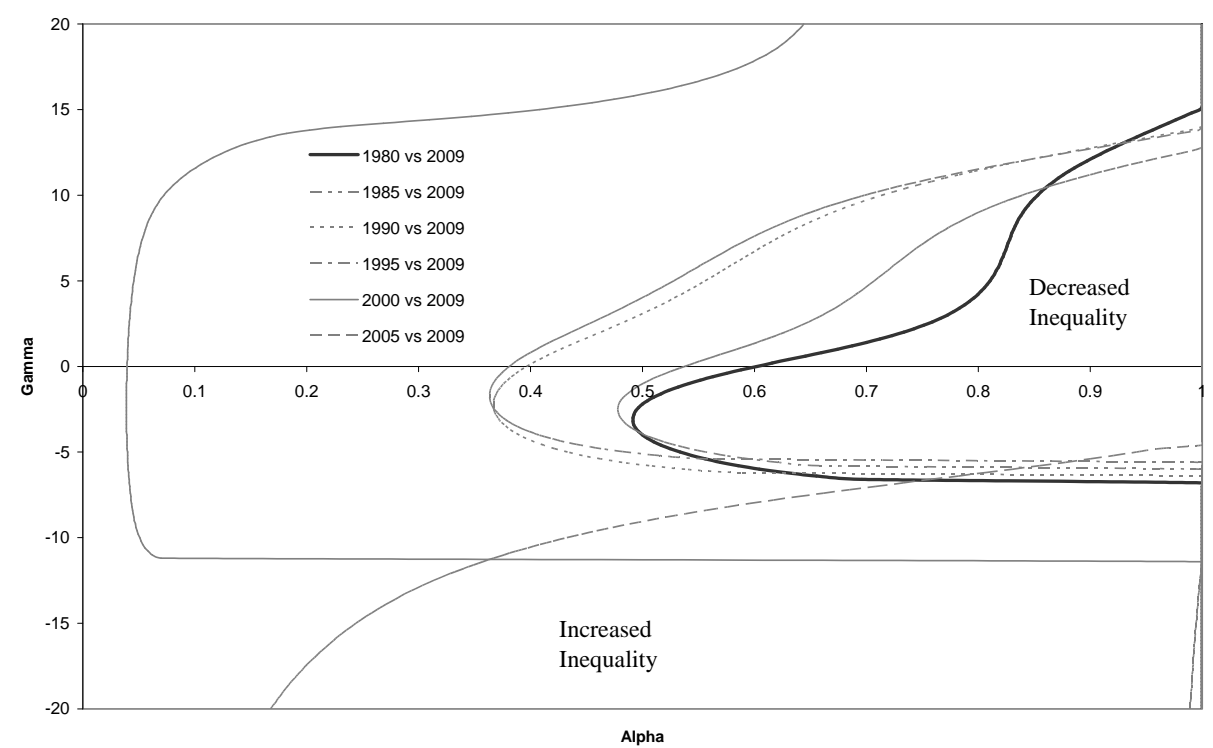

Figure 3: Increasing and decreasing inequality in the $(\alpha, \gamma)$ normative space according to the Bossert and Pfingsten GE-index (Data: World Bank, 2010)

show increased inequality if either $\gamma$ is sufficiently low or sufficiently high. With the exception of the 2005-2009 comparison, this pattern also emerges for the shorter periods of comparison.

\section{Conclusion}

In this paper we applied absolute, relative and intermediate concepts of inequality to the assessment of the evolution of global inequality. We used World Bank (2010) data on population and GDP per capita for 115 countries in the period 1980-2009.

Based on our tests for $\alpha$-Lorenz dominance, we found overwhelming support for the claim that inequality has increased over time. For many of the pairwise comparisons213 out of 435 cases - the income distribution of the earlier year Lorenz dominates that of the later year for the absolute case. That is, all absolute measures indicate an increase in inequality over time for these comparisons. Moreover, we showed that one can usually move substantially in the direction of the relative view without losing the unanimous verdict of increased inequality. Strikingly, the reverse test-whether the year with the larger income dominated the year with the lower income for some minimal level of $\alpha$ did not yield any result. This means that we did not find robust evidence for decreased inequality over the last three decades, neither for the absolute view, nor for the relative view, nor for any view intermediate between the two polar cases. 
We considered absolute, intermediate and relative versions of the generalized entropy class and of the S-Gini class and determined the combinations of the two parameters (local relativeness and bottom-sensitivity) underlying a judgment on the evolution of global inequality. Several popular relative members of these classes indicate that inequality has decreased over time. However, we showed that one does not need to rely on genuine absolute measures to reverse the conclusion. For the changes in the world income distribution over the period 1980-2009, some intermediate level of relativeness (with a value of $\alpha$ around 0.5 ) suffices to produce unanimous agreement on increased inequality within the corresponding subset of inequality measures.

The purpose of this paper was not to provide a single definite answer to the dispute whether world inequality has decreased or increased. On the contrary, we precisely stressed that in a context of income growth an unavoidable normative choice concerns the invariance concept that one adopts. We have shown that the specific choice of invariance concept does indeed have a major impact on the answer one gives to the simple question of whether world inequality increased or not. Increasing absolute gaps may only be a temporary phenomenon if the divergence of growth rates between poor and rich countries remains sufficiently large in the future. This does not, however, in any way diminish the usefulness of revealing normative and methodological choices which can explain apparently opposite scholarly conclusions.

\section{References}

Amiel Y and FA Cowell, 1999a. Income transformations and income inequality. In: D Slottje (ed.) Advances in Econometrics, Income Distribution and Scientific Methodology. Heidelberg: Physica Verlag, 209-232.

Amiel Y and FA Cowell, 1999b. Thinking about Inequality, Cambridge, UK: Cambridge University Press. Anand S and P Segal, 2008. What do we know about global income inequality? Journal of Economic Literature 46, 57-94.

Atkinson AB, 1970. On the measurement of economic inequality. Journal of Economic Theory 2, 244263.

Atkinson AB and A Brandolini, 2004. Global world inequality: absolute, relative or intermediate? Mimeo.

Atkinson AB and A Brandolini, 2010. On analyzing the world distribution of income. World Bank Economic Review 24, 1-37.

Ballano C and J Ruiz-Castillo, 1993. Searching by questionnaire for the meaning of income inequality. Revista Española de Economía 10, 233-259.

Besley TJ and IP Preston, 1988. Invariance and the axiomatics of income tax progression: a comment. Bulletin of Economic Research 40, 159-163.

Bosmans K and FA Cowell, 2009. The class of absolute decomposable inequality measures. Economics Letters 109, 154-156. 
Bossert W and A Pfingsten, 1990. Intermediate inequality: concepts, indices, and welfare implications. Mathematical Social Sciences 19, 117-134.

Bourguignon F, 1979. Decomposable income inequality measures. Econometrica 47, 901-920.

Bourguignon F and C Morrisson, 2002. Inequality among world citizens: 1820-1992. American Economic Review 92, 727-744.

Capéau B and A Decoster, 2005. The rise or fall of world inequality. A spurious controversy? World Economic Papers 166, 37-53.

Chakravarty SR and S Tyagarupananda, 1998. The subgroup decomposable absolute indices of inequality. In: SR Chakravarty, D Coondoo and R Mukherjee (eds) Quantitative Economics: Theory and Practice, Essays in Honor of Professor N. Bhattacharya. New Delhi: Allied Publishers Limited, 247-257.

Chakravarty SR and S Tyagarupananda, 2009. The subgroup decomposable intermediate indices of inequality. Spanish Economic Review 11, 83-97.

Chotikapanich D, R Valenzuela and DSP Rao, 1997. Global and regional inequality in the distribution of income: estimation with limited and incomplete data. Empirical Economics 22, 533-46.

Cowell FA, 1980. On the structure of additive inequality measures. Review of Economic Studies 47, 521-531.

Cowell FA, 2006. Theil, inequality indices and decomposition. Research on Economic Inequality 13, 345-360.

Cowell FA and K Kuga, 1981a. Inequality measurement. An axiomatic approach. European Economic Review 15, 287-305.

Cowell FA and K Kuga, 1981b. Additivity and the entropy concept: an axiomatic approach to inequality measurement. Journal of Economic Theory 25, 131-143.

del Rio C and J Ruiz-Castillo, 2000. Intermediate inequality and welfare. Social Choice and Welfare 17, 223-239.

Donaldson D and JA Weymark, 1980. A single-parameter generalization of the Gini indices of inequality. Journal of Economic Theory 22, 67-86.

Donaldson D and JA Weymark, 1983. Ethically flexible Gini indices for income distributions in the continuum. Journal of Economic Theory 29, 353-358.

Dowrick S and M Akmal, 2005. Contradictory trends in global inequality: a tale of two biases. Review of Income and Wealth 51, 201-229.

Ebert U, 2004. Coherent inequality views: linear invariant measures reconsidered. Mathematical Social Sciences 47, 1-20.

Kolm S-C, 1969. The optimal production of social justice. In: J Margolis and H Guitton (eds.) Public Economics. An Analysis of Public Production and Consumption and Their Relations to the Private Sectors. London: Macmillan.

Kolm S-C, 1976a. Unequal inequalities I. Journal of Economic Theory 12, 416-442.

Kolm S-C, 1976b. Unequal inequalities II. Journal of Economic Theory 13, 82-111.

Krtscha M, 1994. A new compromise measure of inequality. In: W Eichhorn (ed), Models and Measurement of Welfare and Inequality. Heidelberg: Springer-Verlag, 111-120.

Marchant T, 2008. Scale invariance and similar invariance conditions for bankruptcy problems. Social Choice and Welfare 31, 693-707.

Milanovic B, 2002. True world income distribution, 1988 and 1993: first calculations based on household surveys alone. Economic Journal 112, 51-92.

Milanovic B, 2005. Worlds Apart. Measuring International and Global Inequality. Princeton/Oxford.

Moyes P, 1987. A new concept of Lorenz domination. Economics Letters 23, 203-207. 
Pfingsten A, 1986. New concepts of Lorenz domination and risk aversion. Discussion Paper 278, Karlsruhe.

Pfingsten A, 1987. Axiomatically based local measures of tax progression. Bulletin of Economic Research 39, 211-223.

Pinkovskiy M and X Sala-i-Martin, 2009. Parametric estimations of the world distribution of income. NBER Working Paper 15433.

Ravallion M, 2003. The debate on globalization, poverty and inequality: why measurement matters. International Affairs 79, 739-753.

Sala-i-Martin X, 2006. The world distribution of income: falling poverty and ... convergence, period. Quarterly Journal of Economics 121, 351-397.

Seidl C and A Pfingsten, 1997. Ray invariant inequality measures. In: S Zandvakili (ed), Research on Economic Inequality, vol. 7. Greenwich: JAI Press, 107-129.

Shorrocks AF, 1980. The class of additively decomposable inequality measures. Econometrica 48, 613625 .

Shorrocks AF, 1984. Inequality decomposition by population subgroups. Econometrica 52, 1369-1385.

World Bank, 2010. World Development Indicators 2010. Downloaded in July 2010 from http://ddp-ext.worldbank.org/ext/DDPQQ/

Yoshida T, 2005. Social welfare rankings of income distributions: a new parametric concept of intermediate inequality. Social Choice and Welfare 24, 557-574.

Zheng B, 2004. On intermediate measures of inequality. In: JA Bishop and Y Amiel (eds), Research on Economic Inequality, vol. 12. Greenwich: JAI Press, 135-158.

Zoli C, 2003. Characterizing inequality equivalence criteria. Mimeo. 


\section{Appendix. Countries in the data set}

The World Development Indicators (2010) contain data for 212 countries. Only countries for which we have data in all periods are included in our analysis, which reduces the data set to 115 countries. Table 3 gives an overview. This reduced data set contains $86.39 \%$ of the 2009 population. The ten most populated absentees from our data set are the Russian Federation, Vietnam, Ethiopia, Myanmar, Ukraine, Tanzania, Poland, Uganda, Iraq and Afghanistan.

Table 3: Countries in the data set

\begin{tabular}{|c|c|c|c|}
\hline Albania & Dominican Republic & Korea, Rep. & Romania \\
\hline Algeria & Ecuador & Latvia & Rwanda \\
\hline Antigua and Barbuda & Egypt, Arab Rep. & Lesotho & Saudi Arabia \\
\hline Argentina & El Salvador & Liberia & Senegal \\
\hline Australia & Estonia & Luxembourg & Seychelles \\
\hline Austria & Fiji & Madagascar & Sierra Leone \\
\hline Bangladesh & Finland & Malawi & Singapore \\
\hline Belgium & France & Malaysia & South Africa \\
\hline Benin & Gabon & Mali & Spain \\
\hline Bolivia & Gambia, The & Mauritania & Sri Lanka \\
\hline Botswana & Germany & Mauritius & St. Kitts and Nevis \\
\hline Brazil & Ghana & Mexico & St. Lucia \\
\hline Bulgaria & Greece & Morocco & St. Vincent and the Grenadines \\
\hline Burkina Faso & Grenada & Mozambique & Sudan \\
\hline Burundi & Guatemala & Namibia & Swaziland \\
\hline Cameroon & Honduras & Nepal & Sweden \\
\hline Canada & Hungary & Netherlands & Syrian Arab Republic \\
\hline Central African Republic & Iceland & New Zealand & Thailand \\
\hline Chad & India & Nicaragua & Togo \\
\hline Chile & Indonesia & Niger & Trinidad and Tobago \\
\hline China & Iran, Islamic Rep. & Nigeria & Tunisia \\
\hline Colombia & Ireland & Norway & Turkey \\
\hline Comoros & Israel & Pakistan & United Kingdom \\
\hline Congo, Dem. Rep. & Italy & Panama & United States \\
\hline Congo, Rep. & Jamaica & Papua New Guinea & Uruguay \\
\hline Costa Rica & Japan & Paraguay & Vanuatu \\
\hline Côte d'Ivoire & Jordan & Peru & Venezuela, RB \\
\hline Denmark & Kenya & Philippines & Zambia \\
\hline Dominica & Kiribati & Portugal & \\
\hline
\end{tabular}

